\title{
An Empirical Exploration of the Yarn in Big Data
}

\author{
Yusuf Perwej \\ Assistant Professor \\ Dept. of Information \\ Technology \\ Al Baha University, Al \\ Baha \\ Kingdom of Saudi \\ Arabia
}

\author{
Bedine Kerim \\ Assistant Professor \\ Dept.of Information \\ Technology \\ Al Baha University, Al \\ Baha \\ Kingdom of Saudi \\ Arabia
}

\author{
Mohmed Sirelkhtem \\ Adrees \\ Assistant Professor \\ Dept.of Information \\ System \\ Al Baha University, Al \\ Baha \\ Kingdom of Saudi \\ Arabia
}

\author{
Osama E. Sheta \\ Assistant Professor \\ Dept.of Information \\ System \\ Al Baha University, Al \\ Baha \\ Kingdom of Saudi \\ Arabia
}

\begin{abstract}
The growth in population and progression of internet services, data size is getting increased day by day where 105000s of Trillion of data files are there in cloud available in unstructured nature. The coming times of Big Data are rapidly arriving for just about all industries. The Big Data can help in metamorphose major business processes by advisable and correct analysis of accessible data. Big data have also played an essential role in crime discover. Hadoop is opensource software in the form of an extremely scalable and fault tolerant distributed system which plays a very remarkable role in data storage and its processing. The Apache Hadoop Yarn is an open source framework developed by Apache Software Foundation. It is used for nursing Big Data. It endows storage as well as processing functionality. In this paper, we aimed to demonstrate a close look to about Yarn. The Yarn as a usual computing fabric to support MapReduce and another application instance within of the same kind Hadoop cluster. Yarn allow multiple applications to run simultaneously on the coequal shared cluster and assent applications to negotiate resources based on necessity. In the end, we are in a nutshell discuss about the design, development, and current state of deployment of the next generation of Hadoop's computes platform Yarn.
\end{abstract}

\section{Keywords}

Big Data, Yarn, Hadoop, Yarn Scheduler, MapReduce, Yarn Frameworks.

\section{INTRODUCTION}

Look around at the technology we have at present, and it's manifestly coming to the conclusion that it's all about data. Subsequently, not only is the amount of data being [1] originate increasing, but the rate of enhancement is also accelerating. From emails to Facebook posts, from buying histories to web links, there are huge data sets increasingly ubiquitously. The Big data tools frequently enable the processing of data on an enormous scale and at a bottommost cost than foregoing solutions [2] [3]. Apache Hadoop is an open-source software framework that provides enormous data storage and distributed processing of enormous amounts of data. The Hadoop framework provides the tools demand to develop and run software applications. The distributed Hadoop [4] model is designed to effortlessly and economically scale up from single servers to thousands of computer machin, each proposition local computation and storage. Thereupon in a short span of time, Yarn has attained a considerable deal of momentum and acquisition in the big data world [5]. Apache Hadoop Yarn is an open source framework for distributed as well as local storage, analysis and processing of big data on commodity hardware. Today nowaday introduction of Yarn in Hadoop provides [6][7] organizations that are managing big data, with even significant processing speed and scalability. It is possible to stream real-time, process data using various engines, use interactive SQL, manage huge data using batch processing on a single platform. An acronym for Yet Another Resource Negotiator, [8] Yarn in Hadoop extricate an obstruction in the first version of Hadoop MapReduce and [9] detract the strict dependency of Hadoop environments on MapReduce. Apache Hadoop Yarn provides solutions for different kinds of a real time application like social networking data analysis, sensor data analysis, and scientific data analysis. In this survey paper, we review and state-of-the-art of Yarn.

\section{REQUIREMENT FOR YARN}

The Yarn (Yet Another Resource Negotiator) is Hadoop's cluster resource management system. The cluster resource management means managing the resources of the Hadoop clusters and here upon the resources we mean Memory, CPU, etc. The release of Hadoop 2.0, however, [10] Yarn was introduced, which open doors for an emerging new world of data processing occasion. Prior to Hadoop 2, Map Reduce is the only way to process the data in the distributed Hadoop Environment. At the beginning, Hadoop was written exclusively as a MapReduce engine and it runs on a cluster, its cluster management components were also reliably coupled with the MapReduce programming paradigm. The idea of MapReduce and its programming instance, were so deeply ingrained in Hadoop that one could not use it for anything else excluding MapReduce. The MapReduce became the base for Hadoop [3] and as an outcome, the only confirmation that could be run on Hadoop was a MapReduce job, batch processing. In the first instance Hadoop 1, there was a single Job Tracker service that was overloaded with several things such as cluster resource management, restarting fiasco tasks, monitoring TaskTrackers, [5] scheduling jobs, managing computational resources etc. There was definitely a necessity to distinct the MapReduce part and the resource management infrastructure in Hadoop. The Yarn was the first goal to perform this disseverance. The Yarn enables multiple applications to [11] run at the same instant on the same shared cluster and allows applications to negotiate resources based on need. As a result, resource allocation/management is middle to Yarn.

\section{THE DEFICIENCY OF THE MAPREDUCE OR HADOOP 1}

In this spot some use cases where MapReduce and Hadoop 1 does not work very well and we are discussed below in this 
section [12].

\subsection{Inappreciable Scalability}

The JobTracker is performing various tasks and running on a single machine, but other available machines are not being used, consequently resulting in limited scalability.

\subsection{Availability Problem}

In Hadoop 1 JobTracker is the single point of availability by this means if JobTracker fails, all jobs must restart.

\subsection{No Horizontal Scalability}

The Hadoop 1 endorsement single name node and single namespace, limited by name node RAM. Because we have hundreds of data nodes in the cluster, the name node remains all its metadata in memory, so we are limited to a maximum of only $60-110 \mathrm{M}$ files in the exhaustive cluster because of a single name node and single namespace.

\subsection{Trouble with Resource Utilization}

The Hadoop 1 is a concept of a predefined number of map slots and detracts slots for every TaskTrackers. The resource utilization problem occurs because maps slots might be full while detract slots is empty.

\subsection{Sluggish Processing Speed}

In Hadoop 1, with a distributed and parallel algorithm, MapReduce process enormous data sets. The tasks that need to be performed Map and Reduce and, MapReduce requires a lot of time to execute these tasks as a result increasing delay. The data are distributed manner and processed over the cluster in MapReduce, which enlargement the time and detract processing speed.

\subsection{Not High Availability}

In Hadoop 1 name node is a single point of failure. In the absence of namenode the filesystem can't be used. We necessity to manually rescue using secondary name node in case of failure. Correspondingly secondary always lags with that of primary, data loss is indispensable.

\subsection{Trouble in carrying out Real-Time Analysis}

MapReduce is batch driven, if I want to do carry out real time analysis as an alternative of batch-processing. There are several applications which need outcome in real time like fraud detection algorithm. On the contrary, in Hadoop 1, due to narrow coupling these engines cannot run freely.

\subsection{Delay}

With Hadoop, MapReduce framework is relatively slow, in as much as it is designed to support dissimilar format, structure and spacious volume of data. In MapReduce, Map takes a set of data and modify it into another set of data, [12] where individual element are split down into key value pairs and detract takes the output from the map as input and process afore and MapReduce need a lot of time for execution these tasks thereby increasing delay.

\subsection{Uncertainty}

Hadoop only make certain that data job is complete, but it's unable to assurance when the job will be finished.

\subsection{Security}

The Hadoop 1 can be challenging in managing the complicated application. If the user doesn't understand how to enable platform who is managing the platform, your data could be at massive hazard. In storage and network levels [6], Hadoop is absent encryption, which is a crucial point of the matter. Hadoop 1 endorsement kerberos authentication, which is difficult to manage.

\subsection{Hard in Running Message-Passing Method}

This is a stateful procedure that runs on every node of a distributed network. The processes communicate with each other by sending messages, and make changes to state based on the messages they receive. This is not feasible in MapReduce.

\section{ALL THESE DIFFICULTIES RESOLVE WITH YARN}

Keeping in mind that the Yarn framework has dissimilar components to manage the dissimilar tasks, now let's see how it counters the deficiency of Hadoop1 or MapReduce. We know that Yarn has a central resource manager component which manages resources and allocates the resources to the application. The several applications can run on Hadoop via Yarn and all applications could share usual resource management. The Yarn is designed to superior the drawback of too much burden on Job Tracker in Hadoop 1 [11]. Yarn also endorsement multi-tenancy approach. Yarn adds more generic interface to run non-hadoop jobs within the Hadoop framework. The Yarn framework does not have any stable slots for tasks. It endows a middle resource manager which allows you to share various applications through a common resource. Additionally, Yarn uncouple MapReduce's resource management and scheduling competence from the data processing component, enabling Hadoop to support more different processing approaches and a comprehensive array of applications. The Yarn comes as a backward-compatible framework, which means any current job of MapReduce can be executed in Hadoop 2.0.

In security concern of the Hadoop Yarn, we talk about a several layer of defense authorization, authentication, audits. Authentication, it can be in two shapes from first user to service e.g. HTTP authentication or second from service to service. In authorization, Apache Hadoop provides identical to Unix file permission and has an access control list for Yarn. In Audit, Apache Hadoop has audit logs for NameNodes that record [13] file creation and opening. Again Yarn giving it the capability of using Kerberos authentication. In fault tolerance if a Yarn resource manager stop working, it recovers from its own failure by restoring its state from a tenacious store on initialization, it assassination all the containers running in the cluster after the recovery process is finished. As long as when a node manager fails, the resource manager explores it by timing out its heartbeat response, marks all the containers running on that node as an assassination, and apprise the failure to all running application master. If the imperfection is transient, then Yarn node manager will re-synchronize with the resource manager, purifying its local state, and continue. In scheduler Yarn when job appeal for coming into the [14] Yarn resource manager, it evaluates all the resources available and places the job correspondingly. Consequently, it is a monolithic scheduler that makes scheduling verdict and deploy jobs to be scheduled. 


\section{THE EMERGENCE OF HADOOP YARN}

The Yarn therefore turns into a data operating system for Hadoop 2, as it enables various applications to coexist in the identical shared cluster. The Yarn took the cluster resource management potential for the MapReduce system so that new engines could use these general clusters [15] resource management potential. This lightened up the MapReduce system to focus on the data processing part, which it is better and will preferably continue to be so.

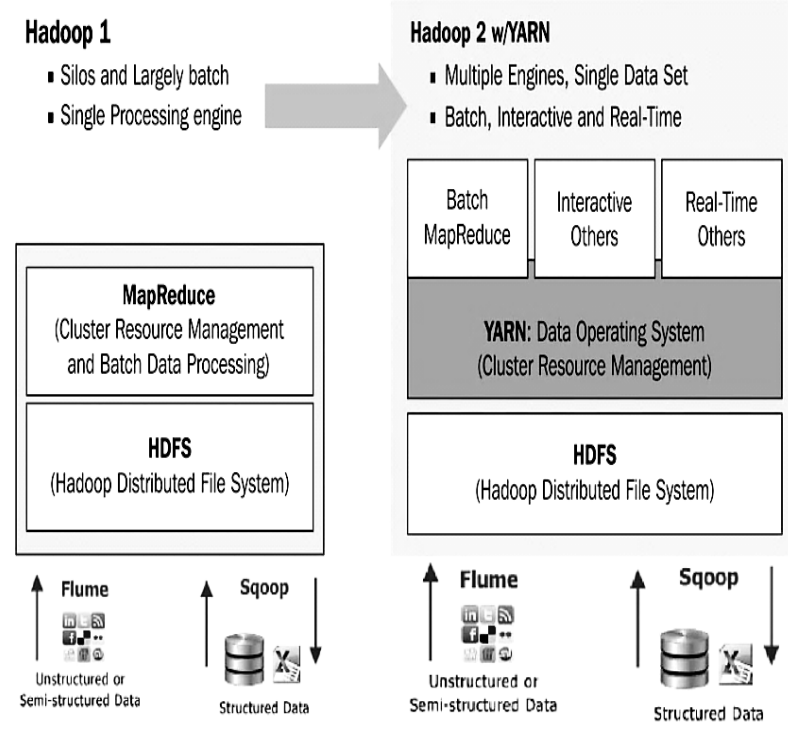

Fig 1: The Emergence of Hadoop Yarn

The Hadoop 1, we are limited to only running MapReduce jobs. The type of work you were performing suitable well into the MapReduce processing model, but it was restrictive for those demands to perform iterative computing, graph processing, [10] or any different type of work. In Hadoop 2 the scheduling pieces of MapReduce were materialize and reworked into a new component called Yarn shown in figure 1 , which is short for Yet Another

Resource Negotiator (Yarn). The basic idea of Yarn is to divided into the functionalities of resource management and job scheduling and monitoring into distinct daemons. The consideration have to global ResourceManager (RM) and [16] per-application ApplicationMaster (AM). Presently applications that wish to operate on Hadoop are implemented as Yarn applications. As an outcome, MapReduce is now a Yarn application.

Eventual Yarn is a general-purpose resource management provision, it is capable of to allocate cluster resources for all kinds of data processing framework implemented for Hadoop. The processing framework, then manages application runtime difficulty more efficiently. Yarn to retain and handle similarity difficulty for all the code that was developed for Hadoop 1, MapReduce role as the [6] initial framework existing for use on Yarn. Yarn provides APIs for requisition and working with cluster resources, but these APIs are not typically used straight from consumer code. Alternatively, a consumer writes to the higher-level APIs provided by distributed computing frameworks, which they are constructed on Yarn and conceal the resource management details from the subscriber.

\section{THE HADOOP YARN ARCHITECTURE}

The Yarn confers a platform to develop and execute distributed processing applications. It also makes better efficiency and resource-sharing capabilities. The Yarn architecture is to support more data [16] processing models, such as Apache Giraph, Apache HAMA, Apache Spark, Apache Storm, and many other, than just MapReduce and Hadoop 1. At this place, we will discuss the high-level architecture of Yarn and look at how the components communicate with each other shown in figure 2. Ours Yarn architecture pursue a master-slave architectural model in which the ResourceManager is the master and node-specific slave NodeManager (NM). The global ResourceManager and per-node NodeManager construct a more scalable, common, and easy platform for distributed application management.

According to Yarn architectural the ResourceManager service runs on the master node of the cluster. A Yarn client proffers an application to the ResourceManager. An application can be a single MapReduce job, manage acyclic graph of jobs, a Java application, or other shell script. The client also explains an ApplicationMaster and a command to commencement the ApplicationMaster on a node. The ApplicationManager handling of the resource manager will validate and accept the application demand from the client. After that scheduler service of resource manager will allocate a container for the ApplicationMaster on a node and the NodeManager ministrations on that node will use the command for beginning the ApplicationMaster ministrations [10][15]. Every Yarn application has a distinctive container called ApplicationMaster.

The ApplicationMaster container is the first container of an application Again NodeManager service runs on every slave of the Yarn cluster. It is in charge of for running application's containers. The resources, identify for a container are taken from the NodeManager resources. Every NodeManager at fixed intervals updates ResourceManager for the set of obtainable resources. The ResourceManager scheduler service uses this resource matrix to allocate latest containers to ApplicationMaster or to beginning execution of a recently developed application.

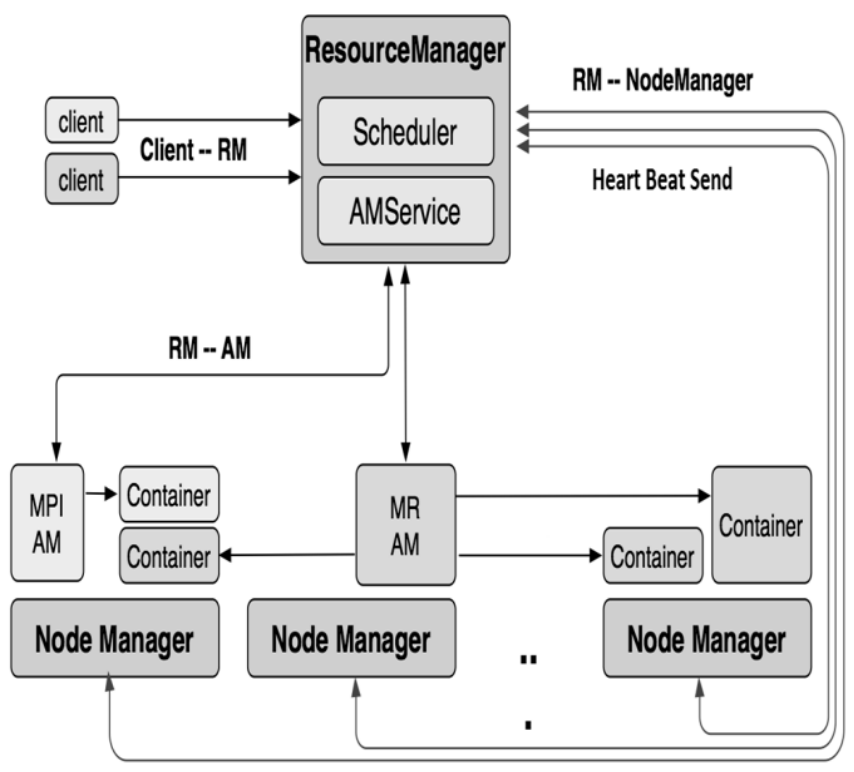

Fig 2: The Architectural View of Hadoop Yarn 


\section{THE BASIC COMPONENTS OF HADOOP YARN}

Yarn brings latest components into the Apache Hadoop workflow. These components provide excellent control for the end-user and at the same time offer more state-of-the-art capabilities to the Hadoop ecosystem. The Yarn splits the amenability of JobTracker into distinct components, ever having a stipulated task to carry out. Yarn splits these amenability of JobTracker into ResourceManager and ApplicationMaster. Practically [16][17] TaskTracker, it uses NodeManager as the worker daemon for implementation of map-reduce tasks. The ResourceManager and the NodeManager form the computational framework for Yarn, and ApplicationMaster is an application conspicuous framework for application management and the fundamental key components of Yarn discussed below in this section.

\subsection{Resource Manager}

The Resource Manager is master that adjudicates all the available cluster resources and thus the assistance manage the distributed applications running on the Yarn system. Resource Manager behaves as a global resource scheduler that is accountable for resource management and scheduling as per the ApplicationMaster's entreaty for the resource need of the application. It is accountable for taking inventory of convenient resources and runs multiple critical services, the most essential of which is the scheduler. The Scheduler component of the Yarn ResourceManager allocates resources to execute applications. This is an example of renovate cluster utilization in terms of CPU cores, fairness, memory etc [17].

\subsection{Resource Tracker Service}

That service is accountable for handling Node Manager registration requests and the at fixed intervals heartbeats every Node Manager dispatch to the Resource Manager. Ahead registration means that a Node Manager denote the Resource Manager that it is obtainable to receive applications and endow information about its at hand CPU cores and physical memory. The mainly two types of service provide firstly to inform the Resource Manager through the Resource Tracker Service of its health and secondly about the present state of the containers at hand in the Node Manager. This information is utilized by the scheduler to update its global view of the cluster resources so that it proficiently allocates them upon ensuing requests from the Application Master.

\subsection{Application Master Service}

The Application master service is accountable for an import type of Yarn services that is a per application service. It is also accountable for negotiating with the Resource Manager to apply resources for a specific application. The entreaty by the Application Masters are handled by the Application Master Service. All entreaty follow the common format and can contain information such as the number of containers [10] demand, resources per container in terms of memory and CPU cores, locality choice and priority of demand from within the application. The application master of the demand to start application, keep an eye on the application progress, and restart, in case of application lack of success.

\subsection{Scheduler}

A scheduler is liable for deciding which tasks get to execute and where and when to execute them. The scheduler is the pivotal service of the Resource Manager. The scheduler has a pluggable strategy plug-in, which is accountable for partitioning the cluster resources among the multiple queues, applications etc. It is only liable for scheduling of tasks and is not worried with status tracking and inquiry of tasks. This concept makes it easy for multiple schedulers to be deployed with the preference being dictated by the needs of the cluster. In addition scheduler does not provide any promise for monitoring or job completion, it only allocates the cluster resources administered by the disposition of job and resource necessity.

\subsection{NodeManager}

NodeManager behave like a per-machine agent and is accountable for managing the life cycle of the container and for monitoring their resource utilization. It's dealing with both the RM and the AM and transmits status of presently running containers and existing resources in terms of memory and CPU on its machine by conveying heartbeats to the RM. It is also liable for terminating containers based on a requisition made by either the RM of the AM. The NodeManager is a per node worker service that is accountable for the execution of containers based on the node competency.

\subsection{Application Master}

The Application Master is at a per-application level and it is liable for the application life cycle management and for parley the suitable resources from the scheduler, keep an eye on their status and progress monitoring. Yarn behaves ApplicationMaster as a third party library responsive for consort the resources from the ResourceManager scheduler and works with NodeManager to run the tasks. It also requisition resource allocation from the scheduler and at fixed intervals reports its status back to the it. It has the accountability of having a talk for convenient resource containers from the Resource Manager, tracking their position, and monitoring progress. Besides, the Application Master can give up a container if the application no longer requires it which disengagement up system resources.

\subsection{Container}

A container is the atomic resource component in Yarn. A container can be understood as rational reservation of resources that will be made use of by task execute in that container. At the basic level, a container is an accumulation of physical resources such as CPU cores, RAM, [18] and disks on a lonely node. A container in Yarn is where a unit of work become in the form of task. A job is devided in tasks and each task gets executed in one container having a particular amount of allocated resources. The clients can cognize container resource requirements when they submit jobs to [19] $\mathrm{RM}$ and execute any variety of applications.

\subsection{Client \& Admin Usefulness}

Yarn endow both client and admin command-line tools. For observation Yarn components, there is a REST API as well as MBeans for daemon processes.

\section{THE FLOW OF A YARN APPLICATION}

In this segment, we are discussing how can move Hadoop Yarn application shown in figure 3.

Move 1 - In move first client submits application lightweight request for an applicationID.

Move 2 - Then move second if the request is well-turned, the application manager will respond with an applicationID. This applicationID will be utilized for the authentic application 
submission to the cluster.

Move 3 - In move third client submits the application to the application manager alongside queue, container strike up commands.

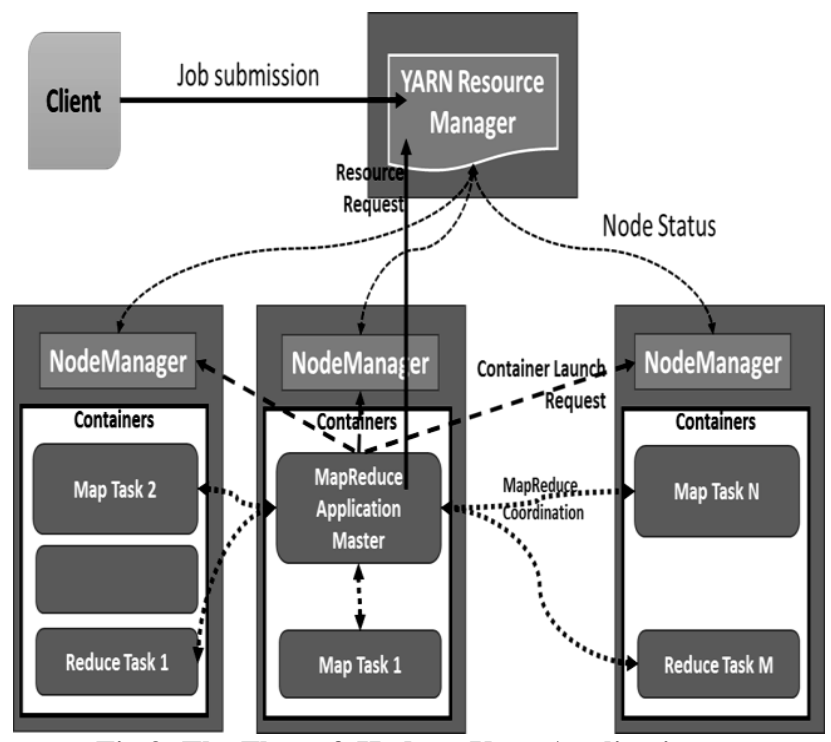

Fig 3: The Flow of Hadoop Yarn Application

Move 4 - Again move fourth application manager is accountable for discovery a container on a node manager to beginning the application master.

Move 5 - Then move fifth as soon as the application master has begun, it will underlie the connection with the resource manager, in particular component called the application master service. It will bring back cluster memory \& CPU availability [10].

Move 6 - In move sixth application master will send a request for containers to execute the application. In a matter of MapReduce, it will inquire for distinguished node managers therefore it wants to ascertain processing with data blocks in the Hadoop distributed file system.

Move 7 - Further move seventh the application master will receive leases on containers per the Yarn scheduler policy. It will also have to care about it enjoyable closing of containers by free its lease to the resource manager[14].

Move 8 - In move eighth as soon as the application master was decided that the application should finish, it can optionally hang on application logs to Hadoop distributed file system, and any other post process activity prior to its self consummate.

\section{THE HADOOP YARN SCHEDULER COMPONENTS}

The Yarn schedulers are the proficient algorithms written to manage cluster resources. The Yarn resource manager service has a pluggable and authentic scheduler component, it does not observe or track the applications running in the cluster. It is answerable only for allocation of resources to executing applications. The scheduling in [17] Yarn is a pluggable framework to allocate cluster resources in a lot of the user environment. Be conditional on the use case and user requirement, administrators may prefer either a simple FIFO scheduler, capacity scheduler, or fair scheduler [16]. Now, we are discussing about the all three scheduler.

\subsection{The FIFO Scheduler}

The native scheduling algorithm that was integrated within the Hadoop version 1 JobTracker was called the FIFO scheduler, explanation first in first out. The FIFO scheduler is fundamentally a straightforward "first come, first served" scheduler in which the JobTracker draw up jobs from a work queue, elderly job first. The FIFO is a queue based scheduler and it is a very straightforward method for scheduling and but it does [20] not assurance the performance efficiency, as every job would use a whole cluster for run. Forthcoming other jobs had to delay in line until running tasks accomplished. This schedule algorithm had no concept of the priority or the size of the job, but the method was straightforward to implement and efficiently.

\subsection{The Capacity Scheduler}

The capacity scheduler is invented to execute Hadoop applications as a shared, multi-tenant cluster in an operator neighborly manner while maximizing the throughput and the make use of the cluster. The capacity scheduling provides invaluable control as well as the efficiency to provide a minimum capacity assurance and share excess capacity among users. The capacity scheduler was developed by Yahoo. Again capacity scheduler imprimatur sharing a cluster while giving every user or group certain minimum capacity assurance. These minimums are not given away with the non appearance of demand. On the inside capacity scheduling, multiple queues are [21] created, every with a configurable number of map and decrease slots. Every queue is also assigned an assured capacity. Suppose if a queue is not consuming its allocated capacity, this surplus capacity for the time being allocated to other queues. Suppose queues can represent a person or massive organization, [22] any obtainable capacity is redistributed for use by other users. There is an added gain that an organization can access any additional capacity not being used by others. This provides elasticity for the organizations in an economical manner. We have configured the capacity scheduler within several Hadoop configuration files. The queues are defined within Hadoopsite.xml, and the queue configurations are set with capacityscheduler.xml. The capacity scheduler works optimal when the workloads are familiar, which assistance in assigning the minimum capacity.

\subsection{The Fair Scheduler}

The Fair scheduler is a third pluggable scheduler for Hadoop that endow an additional way to contribution large clusters. Fair scheduling is a technique of [20] allocate resources to applications such that all applications get, and an equal contribution of resources extra duration. The outcome is that jobs that require short time are able to access the CPU and finish amalgamate with the running of jobs that need more time to run. The fair scheduler was developed by Facebook. The Hadoop implementation makes a set of pools into which jobs are placed for preference by the scheduler. Every pool can be assigned a set of shares to equilibration resources across jobs in pools. All pools have equivalent shares, but configuration is practicable to provide more or diminutive shares be conditional on the job type.

The number of jobs alive at one time can also be constrained, if applicable, to minimize congestion and allow work to finishing in a timely fashion. To make certain fairness, every user is assigned to a pool. Therein, if one user submits several 
jobs, he can obtain the same kind share of cluster resources as all another users. Anyway the shares assigned to pools, if the system is not loaded, jobs obtain the shares that would else divided among the attainable jobs. The fair scheduler allocates a promise least possible share of resources to the pools. This is eternally advantageous for the groups, users, or applications, as they continually get enough resources for execution. The fair scheduler works optimally when there is a lot of mutable between queues.

\section{YARN FRAMEWORKS}

It's the dawn of 2016, and big data is still in its burgeoning stage. Several new startups and giants are investing a spacious amount into developing state-of-the-art frameworks to cater to a new and emerging variety of issues. These frameworks are the modern cutting-edge technologies or programming models that recline to solution the problems [23] across industries in the real world of big data. In this scenario, Yarn endorsement for several programming models and frameworks makes it perfect to be integrated with these new and emerging frameworks. The Yarn forms a resource [10] management platform, which endow services such as fault monitoring, data locality, scheduling, and more to Hadoop 1, Map-Reduce and other frameworks [14], it permits these new application frameworks to attention on extricate the issue that they were specifically meant for. In this below section, we describe briefly following frameworks that run on Yarn.

\subsection{Apache Giraph}

The Apache Giraph is a scalable, fault-tolerant implementation of graph-processing algorithms in Apache Hadoop clusters of up to thousands of computing nodes. Apache Giraph is actual time graph processing software that is for the most part used to analyze social media data. In this scenario, Facebook, Yahoo and Twitter, PayPal all are consumer of Giraph, to help represent and analyze the trillions of connections across large-scale datasets. The Giraph originated as the open-source equivalent to Pregel, the graph processing architecture developed by Google and referred in a 2010. A Giraph algorithm is an iterative execution of masterly steps, that consist of a message swapping phase followed by a concentricity and the node or edge property update phase. During the time that vertices and edges are held in memory, the nodes swapping messages in parallel. Consequently, all worker nodes communicate and send each other small messages, ordinarily of very low data volume. The Giraph's Yarn related abstraction is convenient to extend or use as a template for new projects. Giraph takes benefit of the application master to execute a more inartificial job control, which includes the capacity to spawn and discharge tasks as part of every Bulk Synchronous Parallel step.

\subsection{HOYA ( HBase on Yarn )}

In HBase on Yarn is the Hoya project creates dynamic and flexible Apache HBase clusters on top of Yarn. The Hoya gives a permission to the users deploy distributed applications across a Hadoop cluster, using the Yarn Resource Manager to assign and distribute parts of an application across the cluster. The Hoya keeps an eye on the health of these deployed containers, and react to their lack of success by creating new instances of them. In addition to its endorsement dynamic updates to cluster size, and thawing and freezing the application. Hoya can take a transcendent distributed application, namely Apache HBase and Apache Accumulo and make it a dynamic Yarn application. Hoya is an application to manage and deploy a alive distributed application in a Yarn cluster. The Hoya implements the functionality via Yarn APIs and HBase's shell scripts. Hoya is now competent of executing protracted lived, dynamic applications in Yarn clusters. In Hadoop Yarn cluster manager makes it possible to alter static, one-per-node and cluster-wide services into dynamic, subscriber specific applications. Where every project can execute their personal version. Using HOYA the resource allocation is more elastic, lack of success and scalability make better, and new applications can be developed more comfortably.

\subsection{Dryad on Yarn}

The Dryad provides dependable, distributed computing, on thousands of servers for extensively data parallel applications. A Dryad systems effort to provide a proficient execution environment for execution these jobs, abstracting users away from requiring to handle common distributed computing requirements such as fault tolerance, communication etc. The Dryad was energized by a diversity of data processing systems, including MPP databases, MapReduce, data parallel programs on GPU etc. Its intention to build a system that could express all these variant of computation. The Dryad was structured from the ground up in the absence of a subsidiary resource management or scheduling framework, and few of its characteristics are present in or shared by dissimilar layers of the Hadoop 2 stack like Yarn. In particular, Dryad hopes the specific number of instances of a vertex at execution to be defined at definition at this moment. Further Dryad's architecture incorporates components that do resource management as well as the job management. A Dryad job is synergic by a component called the Job Manager and tasks of a job are run on cluster machines by a Daemon process. Establish communication with the tasks from the job manager become via the Daemon, which acts like a proxy. The Dryad permits one specific optimization via which processing nodes can run at the same time, co-located and connected through pipes or shared memory.

\subsection{Apache Flink}

The Apache Flink is provided for distributed data processing platform for use in big data applications, in the first instance involving analysis of data stored in Hadoop clusters. Apache Flink dataflow programming model provides occurrence at a time processing on both finite and infinite datasets. The basic of Apache Flink is a distributed streaming dataflow engine written in Scala and Java. The Apache Flink architecture shown in figure 4. The Flink execute on Yarn next to other applications. The subscriber does not have to setup or install something if there is beforehand a Yarn setup. Flink provides a low-latency streaming engine, high-throughput, and support for occurrence time processing and state management. In spite of everything Flink is also a strong tool for batch processing. The Flink streaming processes data streams as real streams, i.e., data elements are straightaway pipelined via a streaming program as early as possible they arrive. This permit to perform resilient window operations on streams. Flink is competent of handling delayed data in streams by the use of watermarks. Apache Flink is a stream processing system capable of process row subsequently row in real time. Finally, Flink is make to be a good Yarn citizen and it can execute current MapReduce jobs straight to its running engine. 


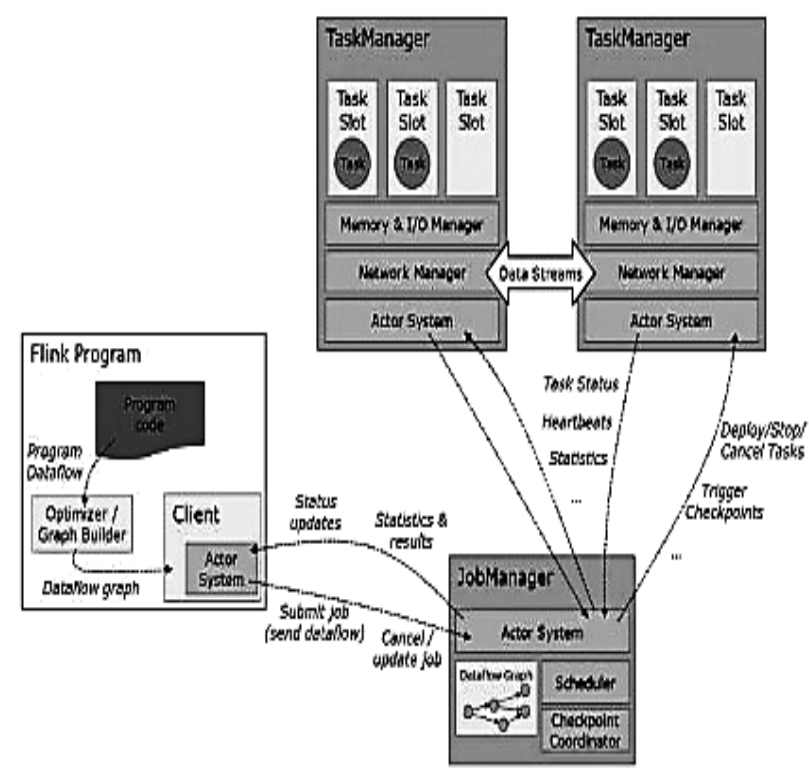

Fig 4: The Apache Flink Architecture

\subsection{Apache Tez}

The Apache Tez is provide an extensible structure for construction exalted performance data processing applications and batch, synergic by Yarn in Apache Hadoop. Apache Tez endow a developer API and framework to write congenital Yarn applications that viaduct the spectrum of interactive and batch workloads. It permits those data penetration applications to work with petabytes of data [24] over thousand nodes. Thereupon Tez component library permits developers to create Hadoop applications that integrate natively with Apache Hadoop Yarn and execution well within combinatorial workload clusters. By the way, Tez also proposition a customizable execution architecture that permits users to express sophisticated computations as dataflow graphs, imprimatur dynamic performance optimizations based on pure information about the data and the resources expected to process it. Tez are not donating directly to end-users, actually it enables developers to build end-user applications with much preferable performance and suppleness. Eventually Tez is constructed on top of Yarn, which is the novel resource-management framework for Hadoop.

\subsection{KOYA ( Kafka on YARN )}

The Yarn has enabled clusters to be used for a heterogeneity of workloads, emerging as a distributed operating system for big data applications. The initiatives are in transit to bring prolonged execution services under the Yarn conservancy, advantage it for centralized resource management and operations. This JIRA is to offer KOYA (Kafka On Yarn), a Yarn application master to initiate and manage Kafka cluster execution on Yarn. Actually the KOYA is a Yarn application that launches Kafka inside Yarn. Eventual after it manages the resource negotiation with Resource Manager, and make certain that Kafka operates in a Yarn native walkway. The Kafka is suitable for the data bus to move data in and exclude Hadoop clusters. Ultimately Kafka's architecture with scalability and preferable performance make it an inartificial fit.

\subsection{Apache HAMA}

The Apache Hama is a distributed computing ideality based on Bulk Synchronous Parallel computing techniques for enormous scientific computations e.g., graph, matrix, and network algorithms. Which was established since 2012 as a high level project of the apache software foundation. In Hama, two types of components are associated with the training procedure first the master task and second the groom task. The master task responsible for merging the model bring up to date information and sending model bring up to date information to all the groom tasks. The groom tasks are responsible for calculating the weight bring up to date pursuant to the training data [25]. The Apache Hama team is glad to declare that we are now accessory not only the Mesos but also the Yarn. The ultramodern Apache Hama endows distributed training of an Artificial Neural Network using its BSP computing engine.

\subsection{Apache Spark}

The Apache Spark is provided an unlocked source cluster computing ideality fundamentally developed at the University of California, Berkeley's AMPLab, the Spark code base was afterwards donated to the Apache Software Foundation. The Apache Spark architecture shown in figure 5. Apache Spark is a rapid, in-memory data processing engine with graceful and meaningful development APIs to permit data workers to proficiently run streaming, machine [26] learning or SQL workloads that need rapidly iterative access to datasets. The Spark executes on Apache Hadoop Yarn, developers ubiquitously can now bring into being applications to exploit Spark power, derive intuition, and elongate their data science workloads within a single, shared dataset in Hadoop. Obviously the Spark assistance to execute an application in Hadoop cluster, up to 110 times intense in memory, and 15 times intense when execute on disk. This is possible by decrease number of read/write operations to disk. It keeps the intermediate processing data in memory. Clearly the spark executes on Yarn in the absence of any pre-installation or root access requisite. It assists to integrate Spark into Hadoop ecosystem or Hadoop stack. It permits other components to execute on the uppermost of the stack.

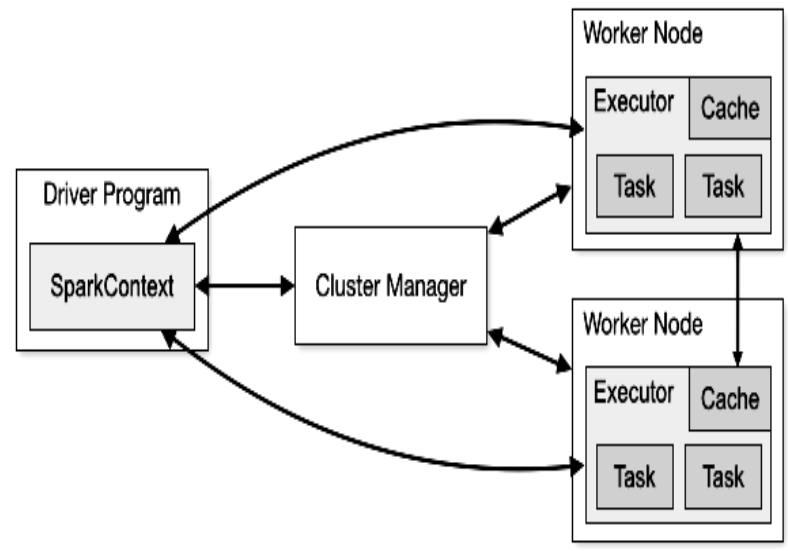

\section{Fig 5: The Apache Spark Architecture 10.9. Hamster ( Hadoop and MPI on the Same Cluster )}

The MPI Project provides for Message Passing Interface execution that is evolving and retain by an industry partner, a consortium of academic, research. The MPI is also provided message passing library specification which defines an enhanced message passing model for parallel, distributed programming on distributed computing environment. The MPI also endows comprehensive, point-to-point, unilateral, 
and parallel I/O communication models. In Point-to-point communications make able swop data between two be similar processes. In comprehensive communication is a simulcast a message from a process for all the another. In unilateral communications make possible remote memory access without correspond to process on the remote node. Three unilateral libraries are obtainable for remote write, remote update and remote read. Therefore, users have whole control of their Yarn containers, there is no cause why MPI applications cannot execute within a Hadoop cluster.

\subsection{Apache S4}

S4 is endowing a distributed, scalable, generic purpose, a pluggable platform that permit programmers to effortlessly develop applications for processing continuous, limitless streams of data. S4 materialize the actors programming instance. The S4 provides state recovery through ill-founded checkpointing. When a node accident, a new node takes over its task and resume from a current snapshot of its state. Events sent after the definitive checkpoint and previous to the recovery are missing. S4 was constructed to be a raised level science neighborly application level structure.

\subsection{Apache Impala}

The Apache Impala is an open source massively parallel processing (MPP) SQL query engine provide for data stored in a computer cluster running Apache Hadoop. Impala endows fast, interactive SQL queries straight on your Apache Hadoop data stored in HDFS, Amazon Simple Storage Service (S3) and HBase. The Impala is therewith to tools at hand for querying big data. Impala does not change the batch processing frameworks construct on MapReduce namely Hive. It implements a distributed architecture based on daemon processes that are accountable for all the facets of query execution that run on the identical machines. Again Impala is integrated with native Hadoop security and Kerberos for authentication, and through the Sentry module, you can make certain that the genuine users and applications are authorized for the genuine data. Eventually Impala is the contain of the columnar storage layout, parquet file format, that is optimized for enormous scale queries typical in the data warehouse.

\subsection{Apache Samza}

The Samza is confer for elastic, real-time, fault-tolerant, asynchronous computational structure for stream processing developed by the Apache foundation in Java and Scala language. The Apache Samza architecture shown in figure 6 . The Samza is a distributed stream processing structure and its utilization a Kafka for messaging, and Hadoop Yarn to endow processor isolation, security, fault tolerance, and resource management. Samza uses Kafka to commit that messages are processed [28] in the order they were written to a echeloned, and that no messages are ever missing. The Samza is echeloned and distributed at every level. Kafka endow ordered, fault-tolerant streams, echeloned, replayable. Yarn endows a distributed environment for Samza containers to execute in. Therewith the Samza function with Yarn, which endorsement Hadoop security model, and resource segregation via Linux CGroups.

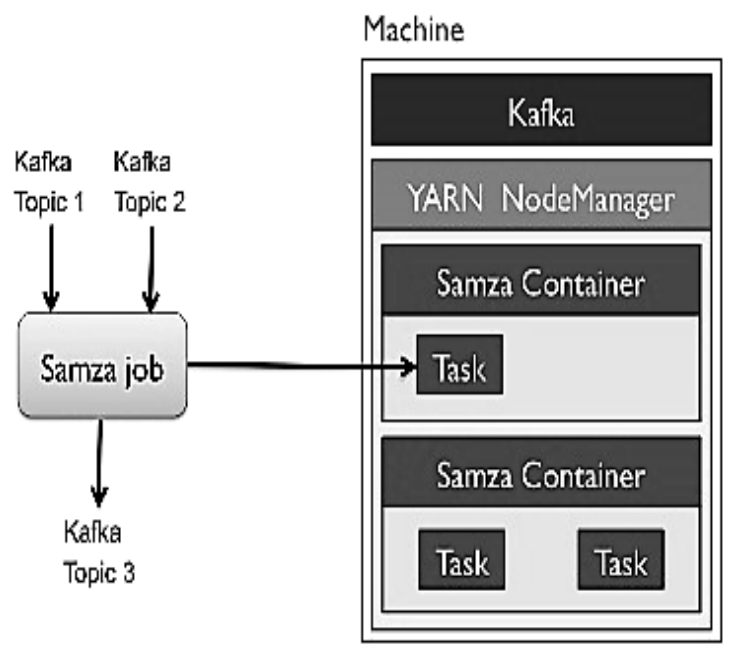

Fig 6: The Apache Samza Architecture

\subsection{Distributed Shell}

The Hadoop Yarn project incorporates the distributed shell application, which is a sample of a non-MapReduce application constructs on top of Yarn. Distributed-Shell is a straightforward mechanism for executing shell commands and scripts in containers on several nodes in a Hadoop cluster. There are several alive implementations of a distributed shell that administrators typically utilized to manage a cluster of machines, and this application is a procedure to show how such a usefulness can be implemented on top of Yarn. The current Distributed-Shell application, you may want to write more intricate logic than merely invoking shell commands. A huge share of the code can be reused with least alteration, assent for fast prototyping of bare-bones Yarn applications.

\subsection{Apache Solr}

The Solr is an enterprise discovery platform, developed in Java, from the Apache Lucene project. Apache Solr is the discovery of data stored in HDFS in Hadoop. The Solr powers the discovery and navigation characteristics of many of the world huge Internet sites, enabling strong full-text discovery and near real-time indexing. Supposing users discover for tabular, text, geo-location or sensor data in Hadoop, they discover it fast with Apache Solr. Solr can be used alongside Hadoop. We know that Hadoop handles a huge amount of data, Solr assistance us in discovering the required information from such a spacious source. Not only discover, Solr can also be used for storehouse intention. For instance, NoSQL databases, it is a non-relational data storage and processing technology. Thereupon Solr provides a comfortable to use, user interface, user neighborly, feature powered, using which we can perform all the practicable tasks example for managing logs, update, delete, add, and explore documents. The Solr is often used to discover text documents and the outcome is delivered according to the relevance to the subscriber query in order.

\subsection{Apache Storm}

Apache Storm is a distributed stream processing, computation structure written primarily in the Java and Clojure programming language. Apache Storm authentic actual time data processing ability to Hadoop. The Apache Samza architecture shown in figure 7. Storm on Yarn is strong for scenarios in need of actual time analytics, machine learning and sustained monitoring of operations. The Storm 
concatenates with Yarn via Apache slider. The Yarn maintains the Storm while also opines cluster resources for data governance, security and functioning components of a contemporaneous data architecture. Principally Storm is conformation to process massive amounts [27] of data in a fault-tolerant and horizontal scalable method. Apache Storm is continuing to be a primate in actual time data analytics. The Storm is convenient to setup, operate and its promise that every message will be processed via the topology at least one time.

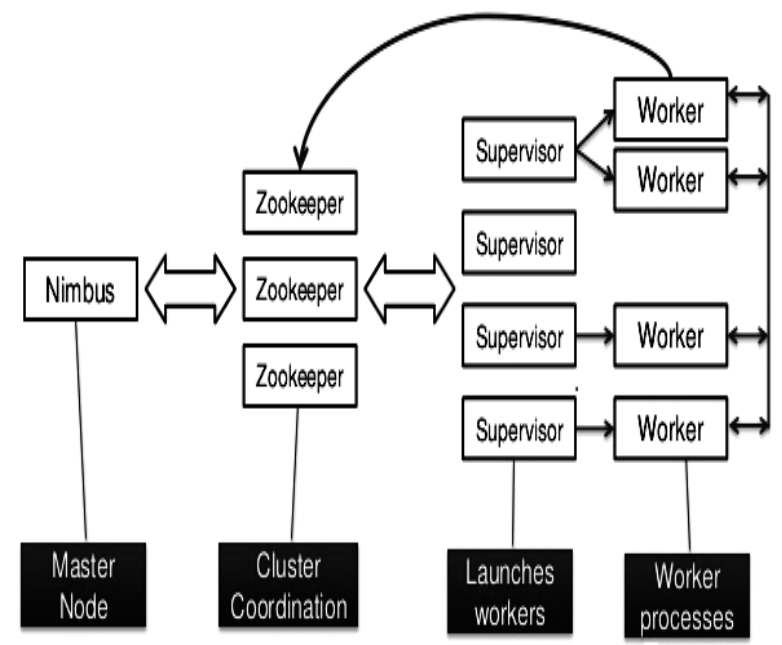

Fig 7: The Apache Storm Architecture

\subsection{REEF ( Retainable Evaluator Execution Framework )}

The Retainable Evaluator Execution Framework (REEF) is a Microsoft project big data technology that affairs with the Hadoop Yarn resource manager and developers can create fault tolerant systems that have the versatility required for changing business needs. Our REEF is a structure to implement scalable, fault-tolerant runtime environments for computational models. With REEF, the client can construct jobs that use data from where it's needed and also maintain state after jobs are done. The structure designers can construction on top of REEF more without difficulty than they can structure directly on Yarn, and can utilize these common services/libraries. REEF endow mechanisms that make easier modality reuse for data caching, and state management absentmindedness that considerably eases the development of elastic data processing work-flows on cloud platforms that support a resource manager service. Finally, REEF is presuming to be well-suited for construction machine learning jobs.

\subsection{Apache Accumulo}

The Accumulo is an extremely scalable classified, distributed key value store basis on Google Bigtable. It is a system construct on top of Hadoop, Apache Thrift and Apache ZooKeeper and developed in Java language. The Apache Accumulo architecture shown in figure 8. It has various novel characteristics like that cell-based access control and a serverside programming mechanism that can ameliorate key/value pairs at several points in the data management process. Accumulo is provide a huge table [29] data storage, lowlatency, and reflow system with cell-level security. It execute on Yarn, the data operating system of Hadoop. Yarn endow visualization [30] and analysis applications with predictable access to data in Accumulo. Every Accumulo key/value pair has its personal security label which limits query outcome based off user authorizations.

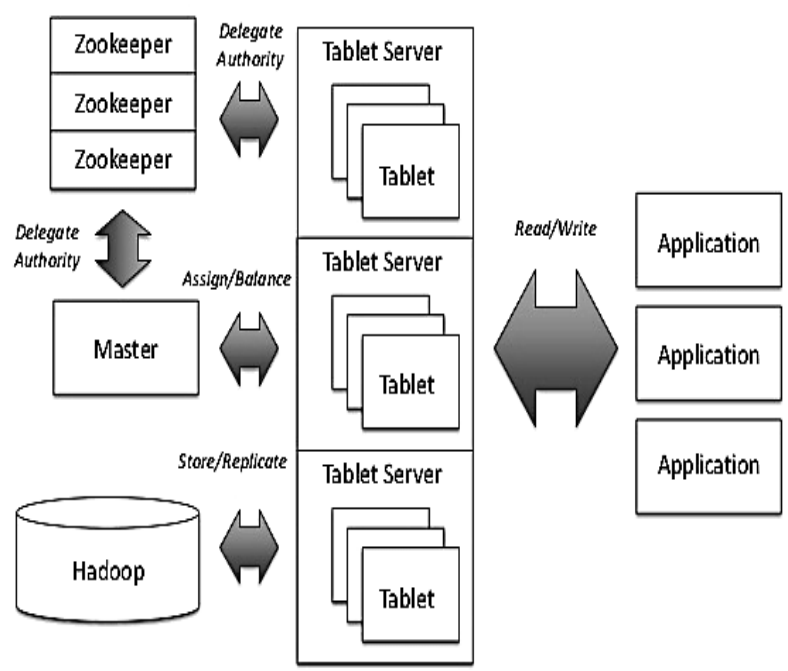

Fig 8: The Apache Accumulo Architecture

\section{CONCLUSION}

In recent times, there has been a growing necessity for computer peripheral device that are capable of handling unprecedented amounts of data. The Big Data mention to all the data that is being originate across the globe at an unprecedented rate as well as this data could be either structured or unstructured. The Apache Hadoop is the most famous and powerful big data tool. It depository massive amount of data in the distributed manner and processes the data in parallel on a cluster of nodes. The Yarn technology lets Hadoop provide enterprise level solutions, beneficence organizations instate preferable resource management. It is a platform for getting compatible solutions, advanced level of security and governing of data over the whole spectrum of the Hadoop cluster. The Yarn is a perfectly rewritten architecture of Hadoop cluster. Hadoop Yarn manages the resources quite proficiently. It's dispense the same on request for any application. Yarn offers straightforward benefit in efficiency, scalability, and flexibility differentiate to the classical MapReduce engine in the initial version of Hadoop. Yarn allocates the cluster resources in a dynamic and efficient manner and due to the make use of is much better compared to prior versions of Hadoop. In this paper, we are presenting deficiency of the MapReduce or Hadoop1, difficulties resolve with Yarn, Yarn concepts, Yarn framework and its components, we also highlight the Hadoop Yarn Architecture. Finally, we investigated the Hadoop Yarn scheduler components. These surveys aim to provide an extensive overview Hadoop Yarn with Big Data.

\section{FUTURE WORK}

For future work, we are planning to analyze classification of Hadoop schedulers and compare the performance of our existing scheduling algorithm in Hadoop environments.

\section{ACKNOWLEDGEMENTS}

The author would like to thank the reviewers anonymous for their constructive comments and that helped whit their revision to improve the resulting quality of this paper. I would like to express my gratitude towards my family and colleges for their co-operation and help me in completing this paper. 


\section{REFERENCES}

[1] Prof. Dr. Philippe Cudré-Mauroux, "An Introduction to BIG DATA", June 6, 2013 Alliance EPFL, http://exascale.info/

[2] S. Kaisler, F. Armour, J.A. Espinosa, and W. Money, Big Data: issues and challenges moving forward, in: Proceedings of the 46th IEEE Annual Hawaii international Conference on System Sciences (HICC 2013), Grand Wailea, Maui, Hawaii, January 2013, pp. 995-1004

[3] Dr. Yusuf Perwej, "An Experiential Study of the Big Data," for published in the International Transaction of Electrical and Computer Engineers System (ITECES), USA, ISSN (Print): 2373-1273 ISSN (Online): 23731281, Vol. 4, No. 1, page 14-25, March 2017. DOI:10.12691/iteces-4-1-3

[4] Hu, H., et. al. (2014). Toward scalable systems for Big Data analytics: A technology tutorial. Access IEEE, 2, 652-687.

[5] “Apache Hadoop," Apache. [Online]. Available: http://hadoop.apache.org/. [Accessed: 18-Oct-2017].

[6] Nikhat Akhtar, Firoj Parwej, Dr. Yusuf Perwej, "A Perusal Of Big Data Classification And Hadoop Technology," for published in the International Transaction of Electrical and Computer Engineers System (ITECES), USA, ISSN (Print): 2373-1273 ISSN (Online): 2373-1281, Vol. 4, No. 1, page 26-38, May 2017. DOI: $10.12691 /$ iteces-4-1-4.

[7] Chen, R., \& Chen, H. (2013). Tiled-MapReduce: Efficient and flexible MapReduce processing on multicore with tiling. ACM Transactions on Architecture and Code Optimization (TACO), 10(1), 3.

[8] Y. Yao, J. Wang, B. Sheng, J. Lin, N. Mi, "HaSTE: Hadoop yarn scheduling based on task-dependency and resource-demand", 2014 IEEE 7th International Conference on Cloud Computing, pp. 184-191, 2014.

[9] Yusuf Perwej, Md. Husamuddin, Fokrul Alom Mazarbhuiya,"An Extensive Investigate the MapReduce Technology "International Journal of Computer Sciences and Engineering IJCSE) E-ISSN: 2347-2693, Volume-5 , Issue-10, Page 218-225, Oct -2017. DOI: $10.26438 / \mathrm{ijcse} / \mathrm{v} 5 \mathrm{i} 10.218225$

[10] Murthy, Arun (2012-08-15). "Apache Hadoop YARN Concepts and Applications". hortonworks.com. Hortonworks. Retrieved 2017-10 -22.

[11] T. C. Bressoud, Q. Tang, "Results of a Model for Hadoop YARN MapReduce Tasks", IEEE International Conference on Cluster Com-nutine, September 2016.

[12] Vasiliki Kalavri, Vladimir Vlassov, "MapReduce: Limitations Optimizations and Open Issues", Trust Security and Privacy in Computing and Communications (TrustCom) $2013 \quad$ 12th IEEE International Conference on, 2013.

[13] Z. Ren, J. Wan, W. Shi, X. Xu, M. Zhou, "Workload analysis implications and optimization on a production hadoop cluster: A case study on taobao", IEEE
Transactions on Services Computing, vol. 7, no. 2, pp. 307-321, April 2012.

[14] Apache. Yarn Scheduler Load Simulator (SLS), [online] Available: http://hadoop.apache.org/docs/r2.7.2/hadoopsls/SchedulerLoadSimulator.html.2016.

[15] V. K. Vavilapalli, A. C. Murthy, C. Douglas et al., "Apache hadoop yarn: Yet another resource negotiator," in Proceedings of the 4th annual Symposium on Cloud Computing. ACM, 2013.

[16] Yi Yao, Jiayin Wang, Bo Sheng, Jason Lin, Ningfang Mi, "HaSTE: Hadoop YARN Scheduling Based on Task-Dependency and Resource-Demand", Cloud'14.

[17] Rostom Mennour, Mohamed Batouche, Oussama Hannache, "MR-SPS: Scalable parallel scheduler for YARN/MapReduce platform", Service Operations And Logistics And Informatics (SOLI) 2015 IEEE International Conference on, pp. 199-204, 2015.

[18] Kebing Wang, Zhaojuan Bian, Qian Chen, "Millipedes: Distributed and Set-Based Sub-Task Scheduler of Computing Engines Running on Yarn Cluster", High Performance Computing and Communications (HPCC) 2015 IEEE 7th International Symposium on Cyberspace Safety and Security (CSS) 2015 IEEE 12th International Conferen on Embedded Software and Systems (ICESS) 2015 IEEE 17th International Conference on, pp. 15971602,2015 .

[19] Yi Yao, Han Gao, Jiayin Wang, Ningfang Mi, Bo Sheng, "OpERA: Opportunistic and Efficient Resource Allocation in Hadoop YARN by Harnessing Idle Resources", Computer Communication and Networks (ICCCN) 2016 25th International Conference on, pp. 1-9, 2016.

[20] M. Isard, V. Prabhakaran, J. Currey et al., "Quincy: fair scheduling for distributed computing clusters", Proceedings of the ACM SIGOPS 22nd symposium on Operating systems principles, pp. 261-276, 2009.

[21] Yi Yao, Jiayin Wang, Bo Sheng, Jason Lin, Ningfang Mi, "HaSTE: Hadoop YARN Scheduling Based on TaskDependency and Resource-Demand", IEEE 7th International Conference on Cloud Computing, pp. 184$191,2014$.

[22] J. Chauhan, D. Makaroff, W. Grassmann, "Simulation and performance evaluation of Hadoop capacity scheduler", 2013.

[23] Apache Hadoop Project, http://hadoop.apache.org

[24] "Apache Software Foundation", Welcome to Apache $\mathrm{Tez}^{\mathrm{TM}}$, Mar 2017, [online] Available: https://tez.apache.org/.

[25] Apache Hama, Jun. 2016, [online] Available: https://hama.apache.org/.

[26] Abdul Ghaffar Shoro, Tariq Rahim Soomro, "Big Data Analysis: Ap Spark Perspective", Global Journal of Computer Science and Technology: C Software \& Data Engineering, vol. 15, no. 1, 2015.

[27] Kenny Ballou, Apache Storm vs. Apache Spark, Apr 
International Journal of Applied Information Systems (IJAIS) - ISSN : 2249-0868

Foundation of Computer Science FCS, New York, USA

Volume 12 - No. 9, December 2017-www.ijais.org $\begin{array}{lrr}\text { 2016, } & \text { [online] } & \text { Available: } \\ \text { http://zdatainc.com/2014/09/apache-storm-apache-spark/ }\end{array}$

[28] Apache samza: Linkedin's real-time stream processing framework, [online] Available: https://engineering.linkedin.com/data-streams/apache- samza-linkedins-real-time-stream-processingframework.

[29] Apache Accumulo. http://accumulo.apache.org

[30] T. C. Bressoud, Q. Tang, Analysis modeling and simulation of Hadoop YARN MapReduce. 